\title{
One-shot multigrid for the optimal Neumann boundary control of time-periodic partial differential equations
}

\author{
Dirk Abbeloos*, Moritz Diehl ${ }^{\dagger}$ and Stefan Vandewalle* \\ *Department of Computer Science, Katholieke universiteit Leuven, B-3001 Leuven, Belgium \\ ${ }^{\dagger}$ Department of Electrical Engineering, Katholieke universiteit Leuven, B-3001 Leuven, Belgium
}

\begin{abstract}
We present a one-shot multigrid method for the optimal Neumann boundary control of time-periodic, linear, parabolic partial differential equations. We focus on optimal control problems of the so-called tracking type, i.e., we try to steer the PDE solution such that it matches a prescribed periodic trajectory, or segment thereof, as closely as possible. To that end we derive the optimality condition of such problems. We develop a one-shot multigrid method for solving the resulting coupled system of forward and backward time-periodic differential equations. Numerical examples are presented to illustrate the behavior of the multigrid algorithm.
\end{abstract}

Keywords: Multigrid, periodic PDE, optimal control

PACS: $82.20 . \mathrm{Wt}, 87.10 . \mathrm{Ed}$

\section{INTRODUCTION}

We consider periodic systems with period $T$, modelled by a linear parabolic partial differential equation (PDE). Our model problem is the one dimensional heat equation, given by

$$
\frac{\partial y}{\partial t}(t, x)-\triangle y(t, x)=f(t, x), \quad t \in[0, T), \quad x \in(0, L)=\Omega,
$$

where $y(t, x)$ will be called the state variable. This equation is combined with a Dirichlet condition on the left, a Neumann condition on the right, and a time-periodicity condition,

$$
y(t, 0)=g(t), \quad \frac{\partial y}{\partial x}(t, L)=u(t) \quad \text { and } \quad y(0, x)=y(T, x) .
$$

We focus on optimal boundary control problems of the so-called tracking type, i.e., we try to steer the PDE solution such that it matches a prescribed periodic trajectory, or segment thereof, as closely as possible. More precisely, we seek to determine the best compromise between the control effort and the difference between the actual state of the system and the desired one, on a subdomain of the space-time domain, or at a particular instance of time. The former cost is expressed by the $L_{2}[0, T]$-norm of the control $u(t)$. For the latter we consider the weighted norm:

$$
\|y-z\|_{w}^{2}=\int_{0}^{T} \int_{\Omega} w(t, x)(y(t, x)-z(t, x))^{2} d x d t,
$$

with $z(t, x)$ the desired state, and $w(t, x)$ a weight function. The optimal control problem is formulated as,

$$
\min _{y, u} J(y, u):=\|y-z\|_{w}^{2}+\alpha\|u\|_{L_{2}[0, T]}^{2},
$$

subject to (1) and (2). The compromise between control effort and approximation error is tuned by the regularization parameter $\alpha$. For example, a small $\alpha$ will yield a good approximation, yet require a large control effort.

Our research on the control of periodic PDEs is motivated by the abundance of such processes in a variety of chemical engineering applications, such as 'simulated moving bed processes' [1] and 'pressure swing adsorbers', which find widespread use in the pharmaceutical and food industry. This article is structured as follows. First we introduce the optimality conditions. Then we overview the multigrid methods used for solving the optimality conditions. In addition we discuss a more specialized problem using a more complex objective function than the one in (4). Finally, we show some numerical results. 


\section{THE OPTIMALITY CONDITIONS}

We adhere to the 'first optimize then discretize' philosophy, i.e., we first state the continuous optimality conditions, and then solve a discretized version of those. The optimal control problem, given by the objective (4) and constraints (1) and (2), is strictly convex. Hence, the first order optimality conditions are sufficient to define a unique solution $\left(y^{*}, u^{*}\right)$. The derivation of the optimality conditions is based on the Lagrangian approach, and involves so-called adjoint variables or Lagrange multipliers, which we denote by $p(t, x)$. Setting all derivatives of the Lagrangian to zero, results in a system of PDEs. The system consists of a set of equations for the states $y(t, x)$ and a set of equations for the adjoint variables $p(t, x)$, as follows

$$
\left\{\begin{array} { l l } 
{ \frac { \partial y } { \partial t } ( t , x ) - \Delta y ( t , x ) } & { = f ( t , x ) } \\
{ y ( t , 0 ) } & { = g ( t ) } \\
{ \frac { \partial y } { \partial x } ( t , L ) } & { = u ( t ) } \\
{ y ( 0 , x ) - y ( T , x ) } & { = 0 , }
\end{array} \quad \left\{\begin{array}{ll}
-\frac{\partial p}{\partial t}(t, x)-\triangle p(t, x) & =w(t, x)(z(t, x)-y(t, x)) \\
p(t, 0) & =0 \\
\frac{\partial p}{\partial x}(t, L) & =0 \\
p(0, x)-p(T, x) & =0,
\end{array}\right.\right.
$$

and is completed with the control equation, $\alpha u(t)-p(t, L)=0$.

\section{A ONE-SHOT MULTIGRID METHOD}

An analytical solution of the optimality system (5) is typically not possible, and so a numerical method is needed. We present an one-shot multigrid method, i.e., the optimality system is solved for the states, multipliers and controls simultaneously. Alternative methods are based on an iterative simulation of the system and repeated update of the controls. The one-shot approach has the advantage of being capable of handling unstable systems.

A multigrid method for the inital value optimal control problem was described in $[2,3]$. This method is extended here to periodic optimal control problems. First, the method of lines with central second order finite differences is applied to discretize the continuous problem, given by (5). The resulting system of ordinary differential equations (ODE) in the unknown vectors $\mathbf{y}$ and $\mathbf{p}$, defined at the spatial grid points, is given by,

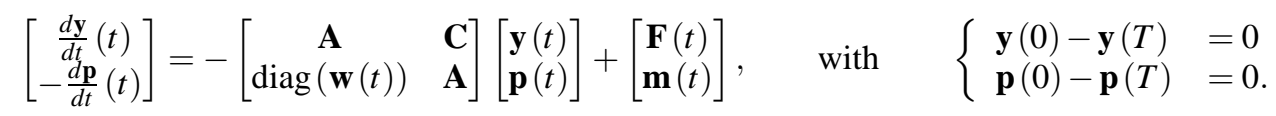

Here, $\mathbf{m}(t)$ is the elementwise multiplication of the vectors $\mathbf{w}(t)$ and $\mathbf{z}(t)$. Matrix $\mathbf{A}$ is the second order discretized diffusion operator $(-\triangle)$, and $\mathbf{C}$ represents the coupling between the states $\mathbf{y}(t)$ and the multipliers $\mathbf{p}(t)$ after eliminating the boundary control $u(t)$. Vector $\mathbf{F}(t)$ collects the right hand side of the PDE and the boundary conditions.

The system of ODEs is solved by using an iterative method of waveform relaxation type, with a multigrid acceleration. The method used is a time-line red-black Gauss-Seidel relaxation. It finds the solution of system (6) by iteratively solving a series of small coupled ODEs for the state $\mathbf{y}_{i}(t)$ and the multiplier $\mathbf{p}_{i}(t)$ gridpoint by gridpoint. The ODEs are discretized with a BDF-1 or BDF-2 method, oriented according to the natural flow of the ODE, i.e., along with the time for $y(t, x)$ and in the opposite direction for $p(t, x)$, as illustrated in Figure 2. The resulting small linear system at each grid point is solved with a band-LU solver.

Because of the slow convergence of the time-line Gauss-Seidel method, the relaxation method is accelerated by using a hierarchy of coarser problems. The multigrid method, given in Algorithm 1, finds the solution of the problem by cycling through the different levels and efficiently combining the approximations on every level. The approximation on level $l \in\{1, \ldots, L\}$ with $L$ the total number of levels in the hierarchy and $l=1$ the coarsest grid, is denoted with $[\mathbf{y}, \mathbf{p}]_{l}$. An approximation is transferred from level $l$ to $l+1$ by using a linear interpolation, and from $l$ to $l-1$ by using a full weighting operator, respectively denoted with $[L]_{l}^{l-1}$ and $[F W]_{l-1}^{l}$. The fully discrete operator corresponding the BDF-discretization of system (6) on level $l$ is denoted with $\mathscr{L}_{l}$.

Algorithm 1 performs satisfactorily when the weight function $w(t, x)$ is of similar magnitude throughout the domain. The algorithm starts to deteriorate, however, when $w(t, x)$ vanishes in large regions. The cause of this has been traced to an instability originating near the right boundary, where the state, controls and multipliers are closely coupled. The encountered problem is similar to solving the biharmonic equation as explained in [4]. The problem was solved by using a blockwise relaxation step at the control boundary. In this step a system of ODEs is solved for the states and the multipliers on the boundary itself and the neighboring internal grid points, as illustrated in Figure 2. 


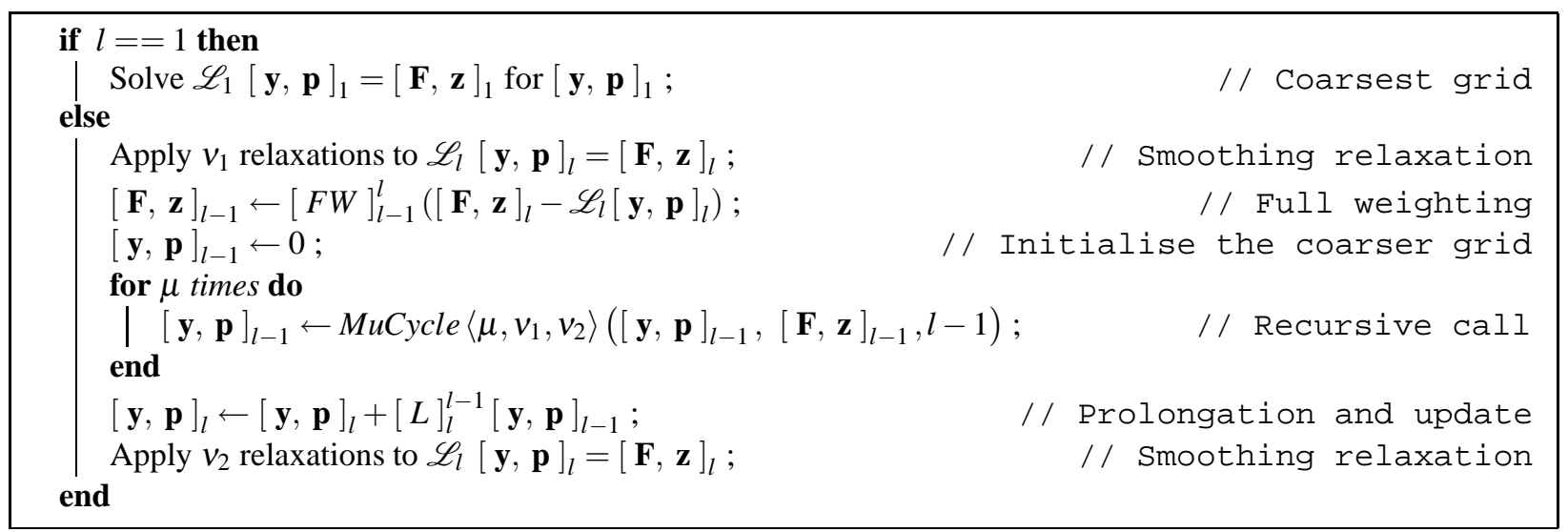

\section{Algorithm 1: $[\mathbf{y}, \mathbf{p}]_{l} \leftarrow$ MuCycle $\left\langle\mu, v_{1}, v_{2}\right\rangle\left([\mathbf{y}, \mathbf{p}]_{l},[\mathbf{F}, \mathbf{z}]_{l}, l\right)$}

\section{A MODIFIED OBJECTIVE FUNCTION}

We also investigated the optimal control problem with the objective (4) replaced by,

$$
\min _{y, u}\left\|\left.y\right|_{t=T}-z\right\|_{L_{2}(\Omega)}^{2}+\alpha\|u\|_{L_{2}[0, T]}^{2}+\beta\left\|\frac{\partial u}{\partial t}\right\|_{L_{2}[0, T]}^{2},
$$

with $\beta$ an additional regularization parameter. Here, we try to minimize the difference between the actual state of the system and a prescribed solution profile $z(x)$ at one particular point in time, i.e., $t=T$. The last term in the new objective (7) represents the cost of the variation of the control $u(t)$. This term is used to reduce the intense oscillating behavior of the solution obtained without this weighting. The optimality conditions for the optimal control problem given by the objective (7) and constraints (1) and (2) consist again of a system of PDEs. This system is composed of the same set of equations for $y(t, x)$, already given in (5), but a different set of equations for $p(t, x)$,

$$
\begin{cases}-\frac{\partial p}{\partial t}(t, x)-\triangle p(t, x) & =0 \\ p(t, 0) & =0 \\ \frac{\partial p}{\partial x}(t, L) & =0 \\ p(0, x)-p(T, x)+y(T, x)-z(x) & =0\end{cases}
$$

and a more complex control equation,

$$
\text { and } \quad \alpha u(t)-\beta \frac{d^{2} u}{d t^{2}}(t)-p(t, L)=0, \quad \text { with } \quad \frac{d u}{d t}(0)=0 \quad \text { and } \quad \frac{d u}{d t}(T)=0 .
$$

The second order ODE in the control $u(t)$ prohibits a simple elimination of the control. The differential equation is discretized by using second order finite differences and it is solved during the relaxation on the control boundary. For $\beta=0$ the shape of the discretized solution depends heavily on the number of gridpoints in the time direction and the value of the regularization parameter $\alpha$. Figure 1 illustrates the dependency on the value of $\alpha$ and on the number of gridpoints used in the time dimension.

\section{NUMERICAL RESULTS}

The table in Figure 3 shows the geometric mean convergence of the residual for the multigrid V(2,2)-cycle for the objective (4) with $w(t, x)=1$ (case 1) and $w(t, x)=w(t)$ (case 2), and the objective (7) (case 3). For case 2, we selected $w(t)=\mathbf{1}_{[0.25,0.5]}(t)$, with $\mathbf{1}_{A}(t)$ the indicator function. That is, the tracking function $z(t, x)$ is to be approximated by the state $y(t, x)$ only on an subinterval of the time-window. The stopping criterium was a relative reduction of $10^{-15}$ of the residual. The plot in Figure 3 shows the optimal control $u(t)$ of model problem (4) with $f(t, x)=0, z(t, x)=3+t \sin (\pi x) \sin (6 \pi t)$ and $w(t)=\mathbf{1}_{[0.25,0.5]}(t)$. 

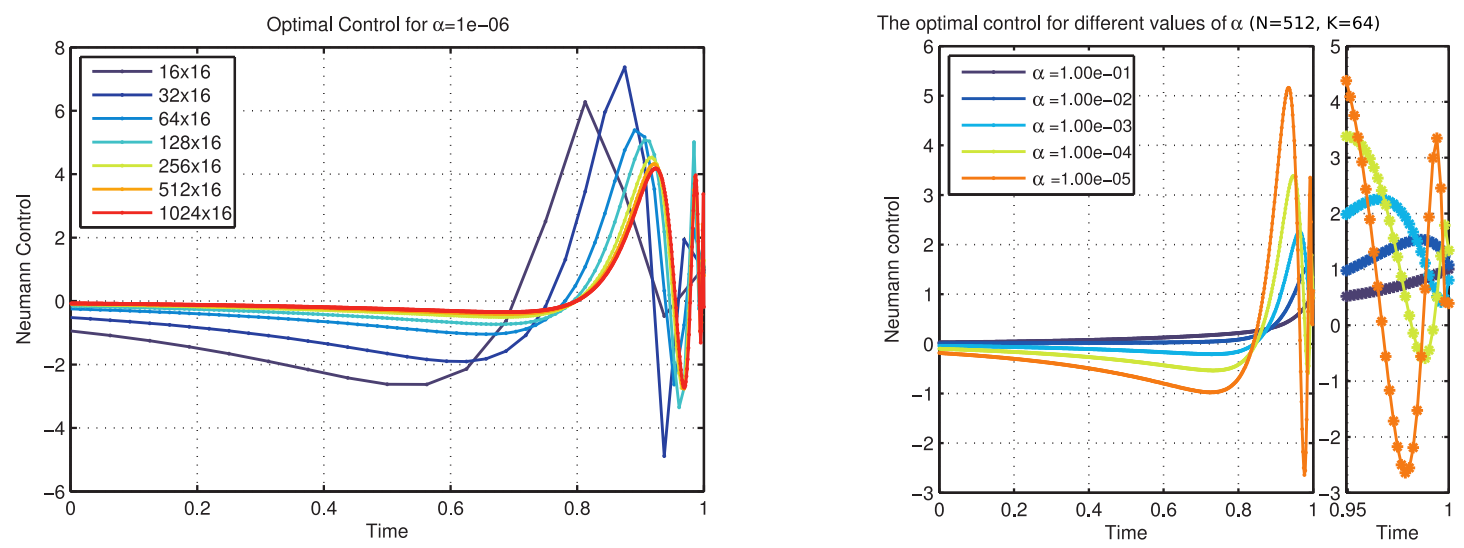

FIGURE 1. Illustration of the dependency of the solution to the discretized optimality conditions on the number of grid points in the time dimension (left), and on the value of the regularization parameter $\alpha$ (right).
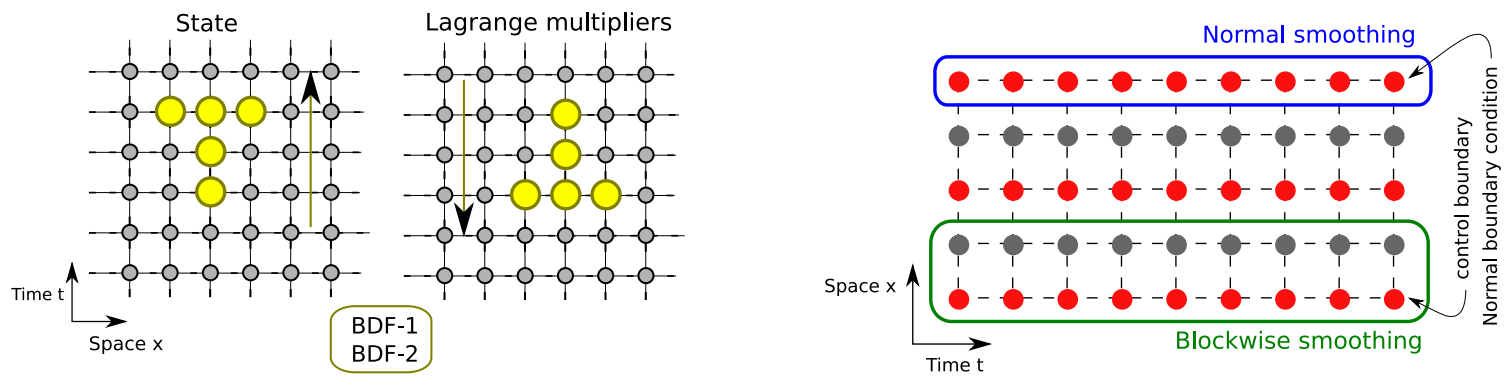

FIGURE 2. The normal and the time-flipped BDF discretisation for respectively the states and the multipliers (left) and an illustration of the blockwise smoothing (right).

\section{REFERENCES}

1. A. Toumi, S. Engell, M. Diehl, H. Bock, and J. Schlöder., Chemical Engineering and Processing 46, 1067-1084 (2007).

2. A. Borzì, Journal of Computational and Applied Mathematics 157, 365 - 382 (2003), ISSN 0377-0427.

3. A. Borzì, and V. Schulz, SIAM Review 51, 361-395 (2009).

4. U. Trottenberg, C. W. Oosterlee, and A. Schuller, Multigrid, Academic Press, 2000, ISBN 012701070X.

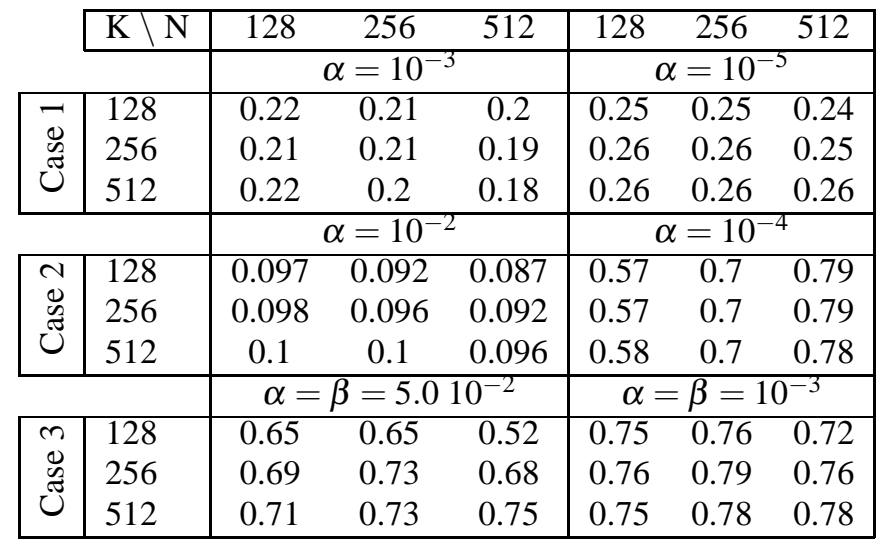

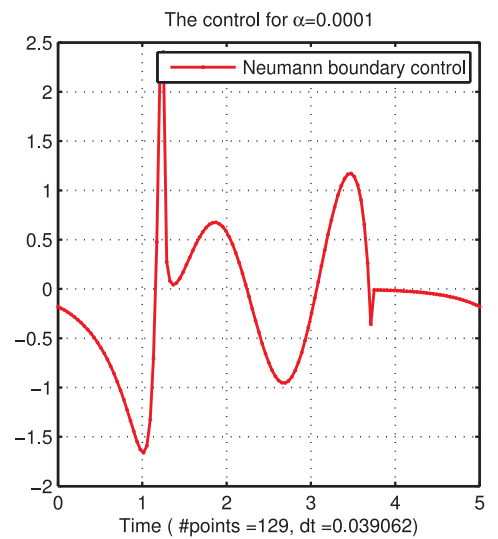

FIGURE 3. The geometric mean convergence of the residual of the multigrid $V(2,2)$-cycle as a function of the number of gridpoints in the time dimension $N$ and in the space dimension $K$, and an example of an optimal control solution $u(t)$. 